\title{
Sustainable Batch Production of Biosynthesized Nanoparticles
}

\author{
Akira Nordmeier, Joseph Woolford, Luke Celeste and Dev Chidambaram*
}

\begin{abstract}
The production of biosynthesized nanoparticles is a slow process with complex isolation and collection procedures due to adhesion to the cell surface. Isolating the microorganism by immobilization in alginate beads will allow for easier recovery of nanoparticles. Immobilized Clostridium pasteurianum was able to reduce Pd ions efficiently through multiple batch production cycles with an average of $99.5 \%$ reduction in the metal ions. Microscopic imaging, diffraction and spectroscopic analyses of the Pd nanoparticles formed using immobilized cultures showed similar size distribution to particles collected from suspended cultures and confirmed the particles were metallic Pd. The Pd nanoparticles also retained their catalytic activity, as shown through the reduction of $450 \mu \mathrm{M}$ methyl orange in less time than the controls. These results demonstrate the effectiveness of the immobilization method for batch production of biosynthesized nanoparticles with little to no difference in particle size and properties.
\end{abstract}

\section{Keywords}

Nanoparticles, Immobilization, Biosynthesis, Alginate

\section{Introduction}

The biosynthesis of nanoparticles (bio-NPs) has accrued interest as a relatively simple, sustainable, and environmentally friendly method for the production of nanoparticles [1]. Biosynthesis processes can make use of plant extracts, bacteria, bacterial extracts, enzymes, and fungi in place of dangerous chemicals [1-5]. Bio-NPs have been shown to exhibit higher catalytic reactivity and greater specific surface area compared to nanoparticles synthesized through chemical methods $[6,7]$. However, more research is needed in order to improve the production and application of biosynthesized nanoparticles. Production rates of bioNPs can be impacted due to toxicity levels from the nanoparticles on the microorganisms at increased

(C) 2017. This manuscript version is made available under the Elsevier user license http://www.elsevier.com/open-access/userlicense/1.0/ 
concentrations [8]. The low production rate can be exacerbated further due to the product lost during the collection method. Through the use of immobilization, the microorganisms can be kept isolated from the nanoparticles, making the separation of microorganisms and Bio-NPs nonessential. The use of immobilization has been shown to eliminate inhibition caused by high concentrations of substrate and product production [9] which, could reduce the toxicity of the nanoparticles. The use of $2 \%$ alginate has the most desirable characteristics and is one of the most widely used methods for whole cell entrapment [10].

This work focuses on the production of nanoparticles through the use of immobilized $C$.

pasteurianum in $2 \%$ alginate beads. The use of alginate beads for the immobilization process should remove the microorganism from the solution without inhibiting the formation of nanoparticles.

\section{Experimental}

All media and solutions were prepared using deionized water $\left(\mathrm{DiH}_{2} \mathrm{O}\right)$ that was boiled for 15 min while being purged with nitrogen gas. All reagents were of analytical grade or better. Clostridium pasteurianum BC1 (ATCC No. 53464) were used in all experiments. Samples of the isolated strain were cultivated under sterile anaerobic batch conditions in a medium consisting of $5 \mathrm{~g} / \mathrm{L}$ yeast extract, $10 \mathrm{~g}$ ammonia chloride, $0.5 \mathrm{~g}$ calcium chloride, $0.3 \mathrm{~g}$ glycerol phosphate, $0.2 \mathrm{~g}$ magnesium sulfate, $0.1 \mathrm{~g}$ peptone, $0.1 \mathrm{~g}$ yeast extract, and $0.003 \mathrm{~g}$ iron sulfate at $\mathrm{pH}$ of 6.8 . The optical density of bacterial solution used was 0.6 determined by measuring bacterial broth using a spectrophotometer at a wavelength of $600 \mathrm{~nm}$. Cell immobilization was performed in an anaerobic glove box with $2 \%(\mathrm{w} / \mathrm{w}) \mathrm{Na}$-alginate solution as discussed in Nordmeier et al. [10]. Beads made from $1 \mathrm{ml}$ of the bacteria/alginate mixture, were then placed into new media vials and incubated for $48 \mathrm{~h}$ prior to use in order reach 20 psi. A Pd stock solution of $1000 \mathrm{mg} / \mathrm{L}$ was made with Disodium tetrachloropalladate. The Pd(II) solution was added to cultures to achieve an initial concentration of $20 \mathrm{mg} / \mathrm{L}$. The media was centrifuged to collect the resulting particulates. The supernatant was then removed for analysis and the particulates were collected for further characterization. New media was then added to the beads, this marked the start of the next cycle. The reusability of the beads was evaluated by 3 cycles of fresh media. The particulates were characterized with samples are characterized transmission electron microscope with an Energy-dispersive X-ray spectroscopy (TEM-EDS, JEOL-JEM 21000F), and powder X-ray powder diffraction (XRD, Rigaku SmartLab diffractometer). The supernatant 
was analyzed using inductively coupled plasma optical emission spectrometry (ICP-OES, PerkinElmer 8000) and a high performance liquid chromatograph (HPLC, Shimadzu LC-20AB) equipped with a refractive index detector (RID-10A) and an Aminex HPX-87H ion exclusion column from Bio-Rad. Cultures with Pd were inoculated with a $10 \mathrm{mM}$ solution of methyl orange (MO) to reach a final concentration of $450 \mu \mathrm{M}$ of MO. The concentration of MO and its degradation product (N,N-Dimethyl-benzene-1,4-diamine) in the culture was monitored spectrophotometrically at $479 \mathrm{~nm}$ and $244 \mathrm{~nm}$ respectively, at $2 \mathrm{~min}$ time intervals as previously described in Johnson et al.[11].

\section{Results and discussion}

\subsection{Bead Regeneration}

The production of acetate, pressure and the glucose consumption was measured at the start and end of each cycle in order to monitor the viability of the immobilized culture. The pressure of the reactors reached on average $21 \mathrm{psi}$ after $48 \mathrm{~h}$ of incubation throughout each cycle (Fig. 1A). The reactors were able to consume approximately $61-75 \%$ of the glucose while producing $0.72-0.81 \mathrm{~g} / \mathrm{L}$ of acetate throughout the cycles, Fig. 1B. The first cycle of the reactors consume the least amount of glucose but was able to produce similar pressure and acetate concentrations as subsequent cycles in the same time frame.
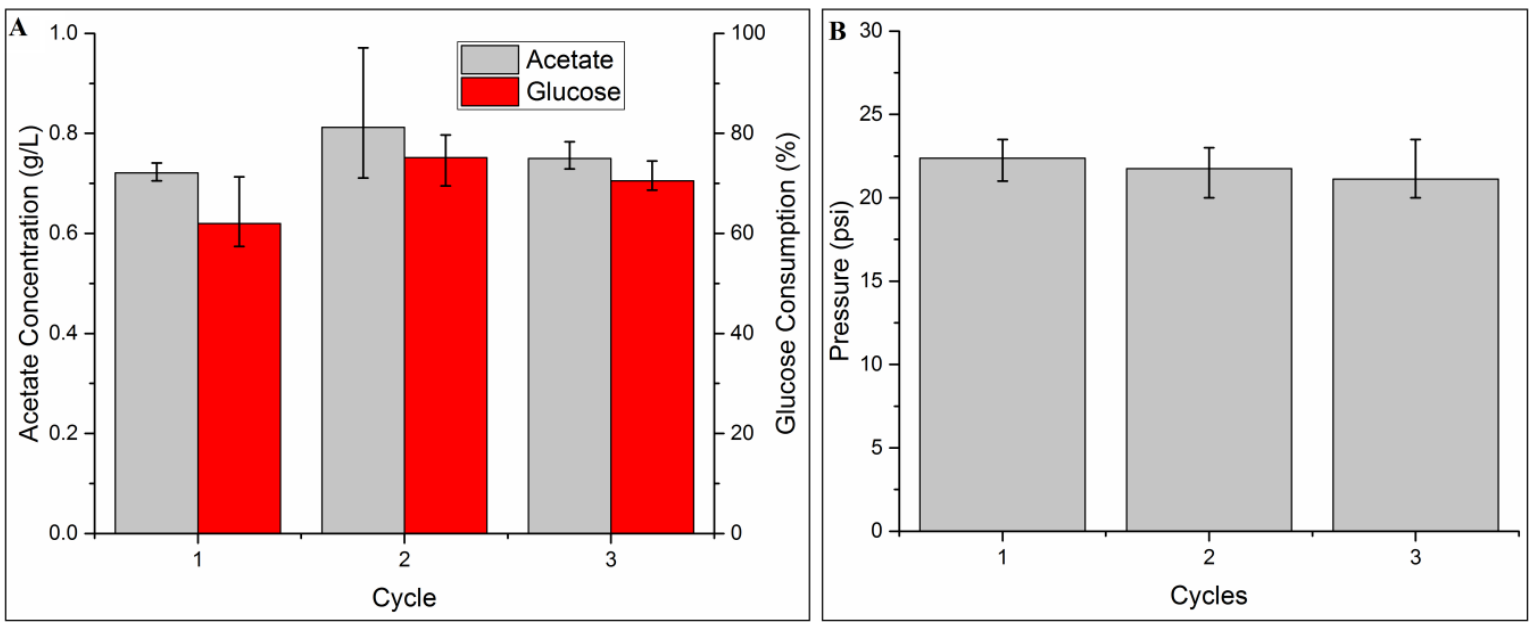

Fig. 1: (A) The pressure of the immobilized batch reactors at time $48 \mathrm{~h}$ for each cycle. (B) The concentration of the acetate that was produced over the time course and the amount of glucose that was consumed by the immobilized bacteria. 


\subsection{Particle formation}

C. pasteurianum is able to produce Pd NPs through a process described previously by one of the authors [12] and this process should not be inhibited by the immobilization technique and therefore the ability of immobilized C. pasteurianum to produce nanoparticles was tested using Pd(II) ions. Immobilized cultures supplemented with Pd(II) had a 99\% Pd reduction which is similar to that of the controls which had 99.7\% removal. The immobilized beads of C. pasteurianum were then transferred to fresh media and allowed to incubate for $48 \mathrm{~h}$ before supplementing with the metal ions. Subsequent cycles of the beads exhibited similar reduction of Pd in all 3 cycles. Three cycles were completed for the biosynthesis of Pd. There was no significant detected cell leakage from the alginate beads during any of the cycles as determined by UV-vis and TEM analysis. The precipitant from cultures supplemented with Pd produced by suspended and immobilized C. pasteurianum was collected for TEM analysis. TEM analysis of the collected samples demonstrated the presence of 2-7 nm size particulates in the precipitant (Fig. 2 A and B). EDS analysis confirmed the presents of Pd in both samples. The size distribution and morphology of the particles collected from the immobilized cultures were comparable to those of the suspended cultures. SEM analysis also corroborates the formation of nanoparticles in both the suspended and immobilized cultures; however, both samples exhibited agglomeration (see Supporting information). XRD analysis of the collected Pd nanoparticles exhibited the diffraction pattern of Pd with a FCC crystal structure, in accordance with JCPDS Card No. 01-088-2335, as shown in Fig. 2C. The particulates produced from the immobilized culture were compared to the particulates produced from the suspended culture. The broadening of the peaks are explained by Chidambaram et al.[12]. 

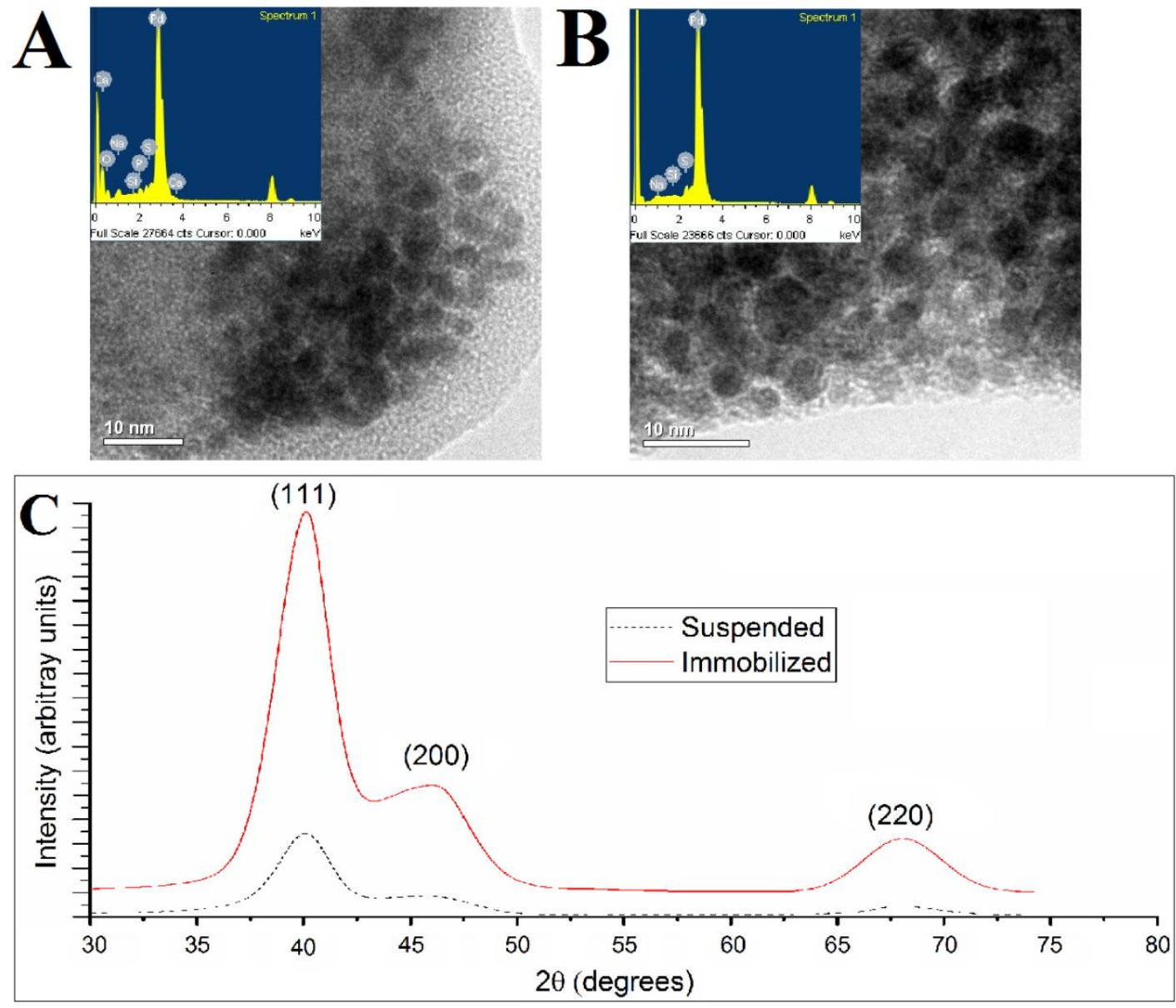

Fig. 2: TEM images of nanoparticles with EDS of the particles shown in panel (A) Pd nanoparticles produced through control culture (B) Pd produced through immobilized culture (C) XRD spectra of suspended bacteria formed Pd nanoparticles (- - ) and immobilized bacteria formed Pd nanoparticles (-).

\subsection{Methyl Orange Degradation}

Immobilized cultures were supplemented with $10 \mathrm{mg} / \mathrm{L} \mathrm{Pd}$ to test the catalytic activity of the $\mathrm{Pd}$ nanoparticles. $10 \mathrm{mg} / \mathrm{L} \mathrm{Pd}$ was chosen for this analysis because at higher Pd concentrations, the degradation occurred too rapidly to determine a rate. Once vials were supplemented with $10 \mathrm{mg} / \mathrm{L} \mathrm{Pd}$, the vials were allowed to sit for 5 min before $450 \mu \mathrm{M}$ of MO was introduced. Samples were collected every 2 min and analyzed by UV-Vis. The $450 \mu \mathrm{M}$ of MO was degraded below the level of detection within 6 min. As shown in Fig. 3, control experiments using heat-killed cells of C. pasteurianum BC1 and Bio-Pd-NPs did not 
degrade MO to any significant extent, while a culture of $C$. pasteurianum without the addition of $10 \mathrm{ppm} \mathrm{Pd}$ was able to reach complete degradation of $450 \mu \mathrm{M}$ MO in $140 \mathrm{~min}$. These results are in agreement with earlier work from this laboratory on degradation of azo compounds by C. pasteurianum [11, 13] and the mechanism behind such degradation. Briefly, the $\mathrm{N}=\mathrm{N}$ (azo) bond is broken down and hydrogenated, leading to the formation of aminated daughter compounds, namely sulfanilic acid and N,N-Dimethylbenzene-1,4-diamine. Sulfanilic acid can be identified using UV-Visible spectroscopy at $244 \mathrm{~nm}$ and was seen in all experiments as reported earlier [11, 13].

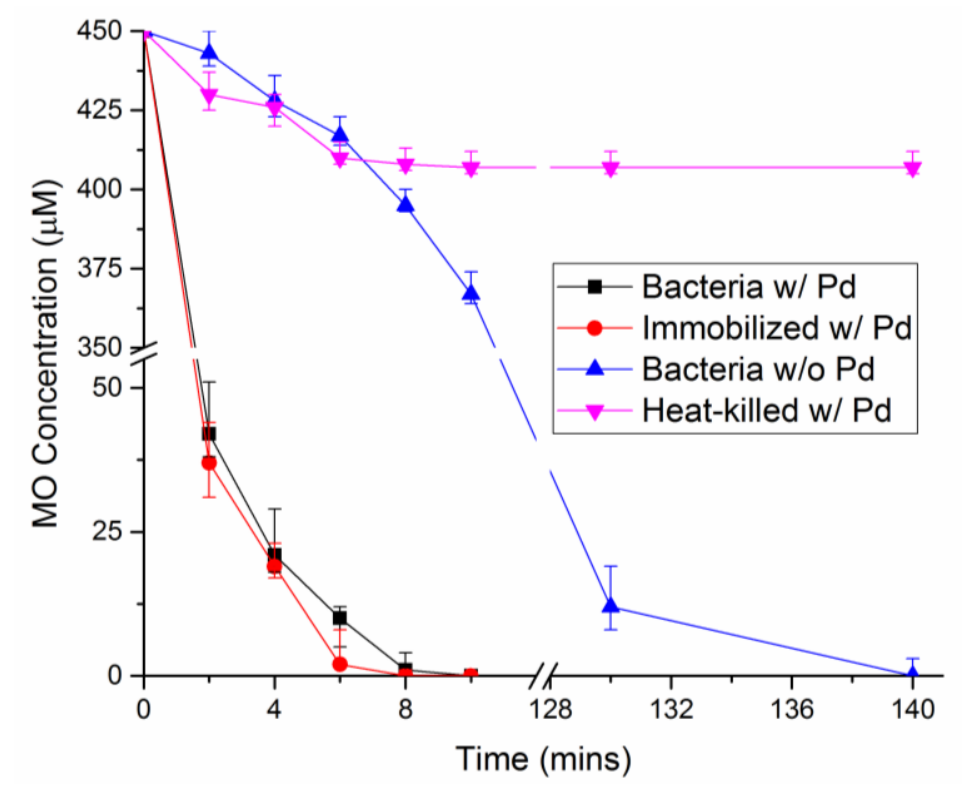

Fig. 3: MO degradation between controls and immobilized cultures supplemented with $10 \mathrm{mg} / \mathrm{L} \mathrm{Pd}$.

\section{Conclusion}

The results reported in this article demonstrate, for the first time, the bioreduction of $\mathrm{Pd}(\mathrm{II})$ ions by immobilized C. pasteurianum. Immobilized C. pasteurianum had comparable reduction of the $\mathrm{Pd}(\mathrm{II})$ ions to that of the control cultures and showed similar physical and catalytic characteristics to those formed in suspended cultures. This supports the use of immobilization for improving the collection of biosynthesized nanoparticles as well as for use in sustainable batch production of nanoparticles. 


\section{Acknowledgements:}

This material is based upon work supported by the National Science Foundation under Grant No. 1159772. We thank Drs. Mojtaba Ahmadiantehrani and Jim Quinn for technical assistance with TEM and SEM, respectively.

\section{References:}

[1] N. Kulkarni, U. Muddapur, Biosynthesis of Metal Nanoparticles: A Review, Journal of Nanotechnology 2014 (2014) 8.

[2] M.N. Nadagouda, N. Iyanna, J. Lalley, C. Han, D.D. Dionysiou, R.S. Varma, Synthesis of Silver and Gold Nanoparticles Using Antioxidants from Blackberry, Blueberry, Pomegranate, and Turmeric Extracts, ACS Sustainable Chemistry \& Engineering 2(7) (2014) 1717-1723.

[3] A.D. Brumbaugh, K.A. Cohen, S.K. St. Angelo, Ultrasmall Copper Nanoparticles Synthesized with a Plant Tea Reducing Agent, ACS Sustainable Chemistry \& Engineering 2(8) (2014) 1933-1939.

[4] S.Y. Lee, S. Krishnamurthy, C.-W. Cho, Y.-S. Yun, Biosynthesis of Gold Nanoparticles Using Ocimum sanctum Extracts by Solvents with Different Polarity, ACS Sustainable Chemistry \& Engineering (2016).

[5] X. Wei, M. Luo, W. Li, L. Yang, X. Liang, L. Xu, P. Kong, H. Liu, Synthesis of silver nanoparticles by solar irradiation of cell-free Bacillus amyloliquefaciens extracts and AgNO3, Bioresour. Technol. 103(1) (2012) 273-278.

[6] X. Li, H. Xu, Z.-S. Chen, G. Chen, Biosynthesis of Nanoparticles by Microorganisms and Their Applications, Journal of Nanomaterials 2011 (2011) 16.

[7] N.I. Hulkoti, T. Taranath, Biosynthesis of nanoparticles using microbes-A review, Colloids and Surfaces B: Biointerfaces 121 (2014) 474-483.

[8] X. Zhang, S. Yan, R.D. Tyagi, R.Y. Surampalli, Synthesis of nanoparticles by microorganisms and their application in enhancing microbiological reaction rates, Chemosphere 82(4) (2011) 489-494.

[9] G.D. Najafpour, Chapter 8 - Immobilisation of Microbial Cells for the Production of Organic Acid and Ethanol, in: G.D. Najafpour (Ed.), Biochemical Engineering and Biotechnology, Elsevier, Amsterdam, 2007, pp. 199-227.

[10] A. Nordmeier, D. Chidambaram, The Influence of Dopants on the Effectiveness of Alginate Beads in Immobilized Cell Reactors, Applied Biochemistry and Biotechnology (2015) 1-7.

[11] A. Johnson, G. Merilis, J. Hastings, M.E. Palmer, J.P. Fitts, D. Chidambaram, Reductive degradation of organic compounds using microbial nanotechnology, Journal of the Electrochemical Society 160(1) (2013) G27-G31.

[12] D. Chidambaram, T. Hennebel, S. Taghavi, J. Mast, N. Boon, W. Verstraete, D. van der Lelie, J.P. Fitts, Concomitant Microbial Generation of Palladium Nanoparticles and Hydrogen To Immobilize Chromate, Environmental Science \& Technology 44(19) (2010) 7635-7640.

[13] A. Johnson, G. Merilis, J. Hastings, M.E. Palmer, J.P. Fitts, D. Chidambaram, Nanotechnology and Microbial Electrochemistry for Environmental Remediation, Electrochemical Society Transactions 33(38) (2011) 103-112. 


\section{Figure Captions:}

Fig. 1: (A) the pressure of the immobilized batch reactors at time $48 \mathrm{~h}$ for each cycle. (B) The concentration of the acetate that was produced over the time course and the amount of glucose that was consumed by the immobilized bacteria.

Fig. 2: TEM images of nanoparticles with EDS of the particles shown in panel (A) Pd nanoparticles produced through control culture (B) Pd produced through immobilized culture (C) XRD spectra of suspended bacteria formed Pd nanoparticles (- - ) and immobilized bacteria formed Pd nanoparticles (-).

Fig. 3: MO degradation between controls and immobilized cultures supplemented with $10 \mathrm{mg} / \mathrm{L} \mathrm{Pd}$. 
